\title{
Determination of Similarity Threshold in Clustering Problems for Large Data Sets
}

\author{
Guillermo Sánchez-Díaz ${ }^{1}$ and José F. Martínez-Trinidad ${ }^{2}$ \\ ${ }^{1}$ Center of Technologies Research on Information and Systems, \\ The Autonomous University of the Hidalgo State \\ Carr. Pachuca - Tulancingo Km. 4.5; C.U \\ 42084 Pachuca, Hgo., Mexico \\ sanchezg@uaeh.reduaeh.mx \\ ${ }^{2}$ National Institute of Astrophysics, Optics and Electronics, \\ Luis Enrique Erro No. 1, Sta. María Tonantzintla, \\ 72840 Puebla, Mexico \\ fmartine@inaoep.mx
}

\begin{abstract}
A new automatic method based on an intra-cluster criterion, to obtain a similarity threshold that generates a well-defined clustering (or near to it) for large data sets, is proposed. This method uses the connected component criterion, and it neither calculates nor stores the similarity matrix of the objects in main memory. The proposed method is focussed on unsupervised Logical Combinatorial Pattern Recognition approach. In addition, some experimentations of the new method with large data sets are presented.
\end{abstract}

\section{Introduction}

In unsupervised Pattern Recognition area many algorithms have been proposed [1] Some of them are based on graph theory. In this paper we will consider the approach based on graph proposed in the Logical Combinatorial Pattern Recognition [2, 3].

In this context, it is assumed that the structure of one universe is not known. To find such structure an initial sample is given, then the problem is precisely to find the classes, the groupings.

The main idea consists in consider the data as vertexes in a graph and the similarity among the objects as edges. In this way the problem of unsupervised classification can be seen as finding subgraphs (clusters) in the initial graph (initial sample).

Note that there exists a natural correspondence among data, their similarity and a graph whose vertexes are objects and the weight of their edges is the similarity between adjacent vertexes.

In this context a parameter $\beta_{0}$ can be introduced for controlling how many similar a pair of objects must be in order to be considered similar. As result then a new graph containing only edges with weight greater or equal than $\beta_{0}$ (the parameter) is obtained.

Therefore depending on the desired closeness in similarity, an appropriate value for this parameter can be chosen by the user and then different graphs are obtained. 
Now the problem is reduced to find in the resultant graph certain subgraphs. For example, we can find connected components in the graph fixing a certain $\beta_{0}$, where each vertex is an object of the sample and all the edges have a weight greater than $\beta_{0}$. Note that when the value of $\beta_{0}$ is modified the graph may change and then the connected components also can change obtaining a different clustering for each value of $\beta_{0}$. Here rises a natural question, what value of $\beta_{0}$ must be chosen?

There are many criteria to find subgraphs (clustering criteria), in [4] are presented some of them as $\beta_{0}$-connected components, $\beta_{0}$-compact sets, $\beta_{0}$-strictly compact sets, and $\beta_{0}$-complete maximal sets.

The problem of choosing an adequate value for $\beta_{0}$, without neither to calculate nor to store a similarity matrix for the objects, is studied in this paper. A new automatic method to obtain a similarity threshold $\beta_{0}$ to generate a well-defined clustering (or near to it) for large data set is proposed. This method is based on the maximum and minimum values of similarity among objects and it calculates an intra-cluster similarity for each cluster. Then the method uses this intra-cluster similarity to obtain a global value, which indicates what clustering has the best average intra-cluster similarity. The new method uses the GLC algorithm [5], which generates a clustering based in connected components criterion for large data sets.

\section{Related Works}

In [6] a method to determine the parameter $\beta_{0}$ for a hierarchical algorithm is proposed. This method uses as similarity between two clusters $C_{i}$ and $C_{j}$ the expres$\operatorname{sion} \beta\left(C_{i}, C_{j}\right)=\max _{\substack{O \in C_{i} \\ O^{\prime} \in C_{j}}}\left\{\beta\left(O, O^{\prime}\right)\right\}$. Then using a traditional hierarchical algorithm, i.e, grouping the two clusters more similar in each level, a dendograme is built. Using the dendograme the user can choose the parameter $\beta_{0}$ that prefers according to the number of clusters generated by this $\beta_{0}$ value.

In this case, the user determines the value of $\beta_{0}$ in function of the number of cluster that he want to get analyzing the dendograme, the method automatically not determine the value $\beta_{0}$.

Another related work is [7], where some concepts and theorems to demonstrate the number of forms that a sample of objects can be partitioned in $\beta_{0}$-connected components, are introduced. The values $\beta_{0}$ that characterize the different forms of partitioning the sample and the cardinality for each component are also studied.

In this paper, an algorithm such that given a number $0<k<m$, (where $m$ is the number of objects in the sample), the $k \beta_{0}$-connected components are generated, is proposed.

Note that this method is very useful if the number of $\beta_{0}$-connected components to form is known, otherwise when the number of clusters to form is an incognita in the problem we can not use the method.

Although these techniques were not developed in order to process large data sets. 
The problem of determining the value of $\beta_{0}$ that generates natural clusters (welldefined clusters) is very important in the context of Logical Combinatory Pattern Recognition approach. Therefore in the next sections a new method to estimate $\beta_{0}$ for large data sets is introduced.

\section{Basic Concepts}

In this section, the context of an unsupervised classification problem in the Logical Combinatorial Pattern Recognition is explained and also some basic concepts that our method uses to determine $\beta_{0}$ are introduced.

Let $\Omega=\left\{O_{1}, O_{2}, \ldots, O_{m}\right\}$ be a set of objects and $R=\left\{x_{1}, x_{2}, \ldots, x_{n}\right\}$ a set of features. A description $I(O)$ is defined for every $O \in \Omega$ and this is represented by an $n$-tuple, e.g. $I(O)=\left(x_{l}(O), \ldots, x_{n}(O)\right) \in D_{l} \times \ldots \times D_{n} \quad$ (initial representation space), where $x_{i}(O) \in D_{i}$; $i=1, \ldots, n$; and $D_{i}$ is the domain of admissible values for the feature $x_{i}$. Di can be a set of nominal, ordinal and/or numerical values.

Hereafter we will use $O$ instead of $I(O)$ to simplify the notation.

Definition 1. A comparison criterion [4] is a function $\varphi_{i}: D_{i} \times D_{i} \longrightarrow L_{i}$ which is associated to each feature $x_{i}(i=1, \ldots, n)$, where:

$\varphi_{i}\left(x_{i}(O), x_{i}(O)\right)=\min \{y\}, y \in L_{i}$, if $\varphi_{i}$ is a dissimilarity comparison criterion between values of variable $x_{i}$, or

$\varphi_{i}\left(x_{i}(O), x_{i}(O)\right)=\max \{y\}, y \in L_{i}$, if $\varphi_{i}$ is a similarity comparison criterion between values of variable $x_{i}$, for $i=1, \ldots, n . \varphi_{i}$ is an evaluation of the similarity or dissimilarity degree between any two values of the variable $x_{i} . L_{i} i=1, \ldots, n$ is a total ordered set, usually it is considered as $L_{i}=[0,1]$.

Definition 2. Let a function $\beta:\left(D_{I} \times \ldots \times D_{n}\right)^{2} \rightarrow L$, this function is named similarity function [8], where $L$ is a total ordered set.

The similarity function is defined using a comparison criterion for each attribute.

Definition 3. In a clustering process (also in supervised classification) will understood by Data set (DS) such collection of object descriptions that the size of the set of descriptions together with the size of the result of the comparison of all object descriptions between objects (similarity matrix) does not exceed the available memory size. A Large Data Set (LDS) will be called in the case when only the size of the set of descriptions does not exceed the available memory size. And a Very Large Data Set (VLDS) will be called when both sizes exceed the available memory size [9].

Definition 4. Let $\beta$ be a similarity function and $\beta_{0} \in L$ a similarity threshold. Then two objects $O_{i}, O_{j} \in \Omega$ are $\beta_{0}$-similars if $\beta\left(O_{i}, O_{j}\right) \geq \beta_{0}$. If for all $O_{j} \in \Omega \beta\left(O_{i}, O_{j}\right)<\beta_{0}$, then $O_{i}$ is a $\beta_{0}$-isolated object.

Definition 5 (Intra_i criterion). Let $C_{1}, \ldots, C_{k}$ be $k$ clusters obtained after apply a clustering criteria with a certain $\beta_{0}$. The intra-cluster similarity criterion (Intra_ $i$ ) is defined as follows: 


$$
\text { Intra_i }\left(C_{i}\right)= \begin{cases}\max _{s} & \text { if } \max _{C_{i}}=\min _{C_{i}}=\max _{s} \\ \max _{C_{i}}-\min _{C_{i}} & \text { if } \max _{C_{i}} \neq \min _{C_{i}} \\ \min _{s} & \text { if } \max _{C_{i}}=\min _{C_{i}} \neq \max _{s}\end{cases}
$$

where $\max _{s}$ and $\min _{s}$ are the maximum and minimum similarity values that $\beta$, the similarity function, can takes (i.e. $\max _{s}=1.0$ and $\min _{s}=0.0$, if $L=[0,1]$ for example). Besides, $\max _{C i}$ and $\min _{C i}$ are the maximum and minimum similarity values among objects belonging to the cluster $C_{\mathrm{i}}$.

According to Han and Kamber [10] a good clustering method will produce high quality clusters (well-defined clusters), with high intra-cluster similarity and low intercluster similarity.

The proposed Intra_ $i$ criterion was inspired in two facts. First, The Intra_ $i$ criterion gives a low weight to those clusters where the difference between the maximum and the minimum similarity between objects is low (or null). Also, this criterion gives a high weight to those clusters formed by only one object. This is because these clusters may be outliers or noise, and its global contribution can generates not adequate results.

Second, in this approach of unsupervised classification based in Graph Theory there are two trivial solutions: when $\beta_{0}=\min _{s}$, obtaining one cluster with all objects of the sample, and when $\beta_{0}=\max _{s}$, generating $m$ clusters, each one formed by only one object.

Then, while $\beta_{0}$ is increased from 0.0 to 1.0 the Intra_ $i$ takes several values in function of the difference between the maximum and the minimum similarity between objects in the different clusters for each clustering. Therefore, we propose that a reasonable way to determine an adequate value of $\beta_{0}$ (associated to a well-defined clustering) is considering a clustering (a set of clusters) with minimum average intracluster similarity.

\section{The Method for Determining $\beta_{0}$}

In this section, we describe the proposed method to obtain a threshold $\beta_{0}$ such that a well-defined clustering is associated to this value.

\subsection{Description of the Method}

The proposed method works in the following way. First, the threshold $\beta_{0}$ is initialized with the minimum value of similarity between objects $\left(\beta_{0}=0.0\right)$. After this, in order to generate several clustering, the method handles a loop, which increases the threshold value with a small constant (INC) and then, a clustering using the GLC algorithm is generated with this $\beta_{0}$ value. For each cluster in a clustering, the maximum and minimum values of similarity among objects are calculated and the Intra_ $i$ criterion is computed. Finally, the method calculates the average Intra_ $i$ for each clustering and takes the minimum value obtaining the threshold $\beta_{0}$ that generates a well-defined clustering in the data set. This process continues until INC reaches the maximum similarity value $\beta_{0}=1.0$. 
The increase value (INC) for $\beta_{0}$ can takes several values, depending of the accurate required in the problem $(0.1,0.15,0.5,0.01$, etc., for example). Two ways to handle this parameter in our method are proposed. The first option simply consists in increase INC until reaches the maximum similarity value. The second option is proposed for similarity functions that depend on comparison functions for features $\left(\varphi_{t} t=1, \ldots, n\right)$ that have the form

$$
\begin{aligned}
& \beta\left(O_{i}, O_{j}\right)=\left\{x_{t} / x_{t} \in R, x_{t}\left(O_{i}\right), x_{t}\left(O_{j}\right) \text { are similar according to } \varphi_{t}\right\} / n \text { or } \\
& \beta\left(O_{i}, O_{j}\right)=\sum_{x_{t} \in R} \varphi\left(x_{t}\left(O_{i}\right), x_{t}\left(O_{j}\right)\right) / n
\end{aligned}
$$

where $n$ is the number of attributes. In this case, the value of INC is fixed as $\mathrm{INC}=1.0 / n$, if $\beta_{0} \in[0.0,1.0]$. In this way, the values for $\beta_{0}$ among $1.0 / h$ and $1.0 /(h+1)$, $h=1, \ldots, n-1$ do not generate any change in the clustering.

The method proposed in this paper is the following:

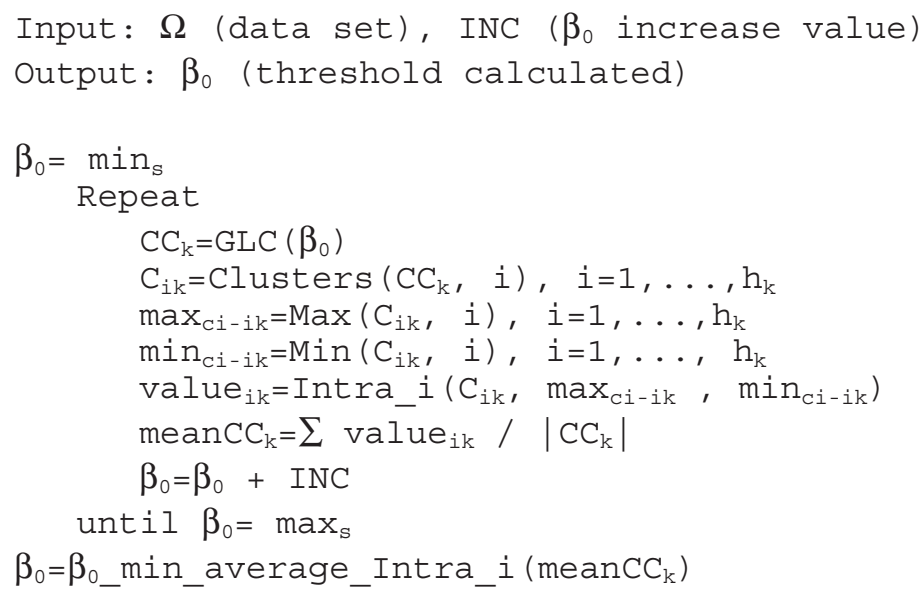

The GLC $\left(\beta_{0}\right)$ function calls to GLC algorithm in order to build a clustering (i.e. $\mathrm{CC}_{k}$ ) with a specific similarity threshold $\beta_{0}$, applying the connected components criterion. The function Clusters $\left(\mathrm{CC}_{k}, i\right)$ returns the cluster $i$, from the clustering $\mathrm{CC}_{k}$. The functions $\operatorname{Max}\left(\mathrm{C}_{i k}, i\right)$ and $\operatorname{Min}\left(\mathrm{C}_{i k}, i\right)$ return the maximum and minimum values of similarity among objects for the cluster $i$, in the clustering $\mathrm{CC}_{k}$. The Intra_i $\left(\mathrm{C}_{i k}, \max _{c i-i k}\right.$, $\min _{c i-i k}$ ) function calculates and return the intra-cluster criterion for the cluster $i .\left|C_{k}\right|$ denotes the number of clusters in a clustering. Finally, $\beta_{0 \_}$min_mean_Intra_i $\left(\right.$meanCC $_{k}$ ) obtains and return the minimum average value of the Intra_ $i$ criterion.

The threshold $\beta_{0}$ obtained by the method indicates that the clustering associated to $\beta_{0}$ is a well-defined clustering. 


\section{Experimental Results}

In this section, two examples of applications of the method to data sets and large data sets are presented.

The first data set (DS1) contains 350 numerical objects in 2D, and it is shown in figure 1(a). The clusters shown in this figure have several shapes, sizes and densities, and they are not lineally separable. The method was applied to DS1 and 7 clustering were obtained. The thresholds $\beta_{0}$ obtained in each clustering $\left(\mathrm{CC}_{\mathrm{i}}\right)$ are as follows:

$\mathrm{CC}_{1}: \beta_{0}=0.00 ;$ No. Clusters $=1$; Average Intra_i=0.8631;

$\mathrm{CC}_{2}: \beta_{0}=0.89 ;$ No. Clusters $=2$; Average Intra_i=0.4777;

$\mathrm{CC}_{3}: \beta_{0}=0.90$; No. Clusters $=3$; Average Intra_i=0.3095;

$\mathrm{CC}_{4}: \beta_{0}=0.93$; No. Clusters $=4$; Average Intra_i=0.2772;

$\mathrm{CC}_{5}: \beta_{0}=0.94$; No. Clusters $=5$; Average Intra_i=0.2378;

$\mathrm{CC}_{6}: \beta_{0}=0.98 ;$ No. Clusters $=86$; Average Intra_ $i=0.6418$;

$\mathrm{CC}_{7}: \beta_{0}=0.99$; No. Clusters $=350$; Average Intra_ $i=1.0000$;

The minimum value of these averages determines the value $\beta_{0}=0.94$, which corresponds to a well-defined clustering (i.e. $\mathrm{CC}_{5}$ ), formed by the clusters shown in the figure 1(b). The same well-defined clustering was obtained in [5]. For this example, the value $\mathrm{INC}=0.01$ was employed.

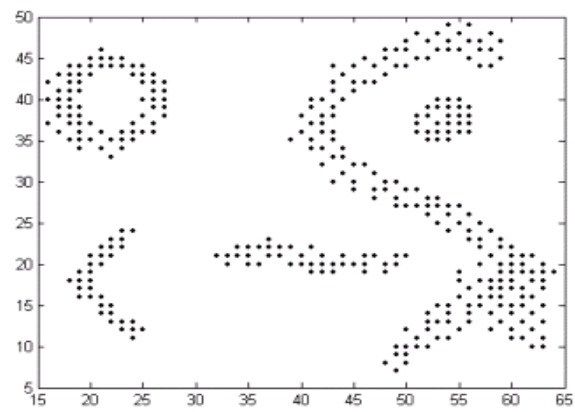

(a)

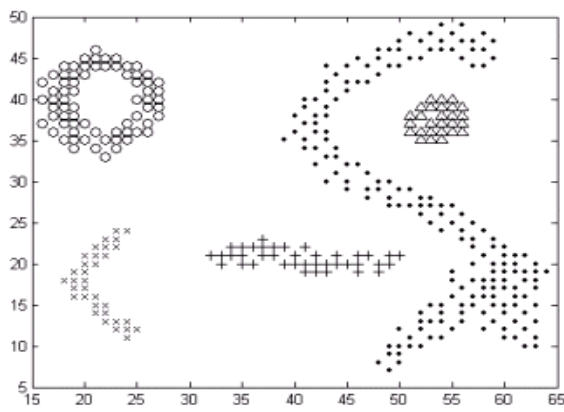

(b)

Fig. 1. (a) The objects corresponding to DS1; (b) Clustering obtained with $\beta=0.94$, for DS1 (well defined clustering discovered)

The second data set used for experimentation was a Mushroom database [11]. The mushroom data set (a LDS, according to our definitions) contains records with information that describes the physical characteristics of a single mushroom (e.g. color, odor, shape, etc.). This data set contains 8124 records. All attributes are categorical, and contain missing values. Each record also contains a poisonous or edible label for the mushroom.

In order to show the behavior of the proposed method, several clustering were obtained with their respective $\beta_{0}$ and them are presented in tables 1 and 2. Again, we show the well-defined clustering generated for DS2, which corresponds with a 
$\beta_{0}=0.9545$ value, generating 23 clusters, with an average Intra_ $i$ value (AIV) of 0.2115 . The same well-defined clustering was obtained in [9]. For this experimentation the value $\mathrm{INC}=0.0454=1.0 / 22$ (number of features $=22$ ) was used.

The cases with $\beta_{0}=0.00, \mathrm{AIV}=0.8182$ and $\beta_{0}=0.99, \mathrm{AIV}=1.0$ are not shown, because in the first case the clustering obtained has all the objects. And, for the second case each cluster contains only one object.

The notation handled in tables 1 and 2 is as follows: $\mathrm{CN}$ denotes the cluster number; NE indicates the number of edible mushrooms; NP denotes the number of poisonous mushrooms, and AIV indicates the average Intra_ $i$ value.

The experiments were implemented in $\mathrm{C}$ language on a personal computer with Pentium processor at $833 \mathrm{Mhz}$ and 128 RAM Megabytes.

Table 1. Clusters obtained for DS2 with $\beta_{0}=0.6810 ; \beta_{0}=0.7273$; and $\beta_{0}=0.7727$;

\begin{tabular}{rrrrrrrrr}
\hline \multicolumn{2}{c}{$\beta=0.6810, \mathrm{AIV}=0.3788$} & \multicolumn{3}{c}{$\beta_{0}=0.7273, \mathrm{AIV}=0.2818$} & \multicolumn{3}{c}{$\beta_{0}=0.7727, \mathrm{AIV}=0.3182$} \\
\multicolumn{1}{c}{$\mathrm{CN}$} & $\mathrm{NE}$ & $\mathrm{NP}$ & $\mathrm{CN}$ & $\mathrm{NE}$ & $\mathrm{NP}$ & $\mathrm{CN}$ & \multicolumn{1}{c}{$\mathrm{NE}$} & $\mathrm{NP}$ \\
\hline 1 & 4016 & 3880 & 1 & 4016 & 2576 & 1 & 392 & 808 \\
& & & & & & & 0 & \\
2 & 192 & 0 & 2 & 0 & 1296 & 2 & 0 & 1296 \\
3 & 0 & 36 & 3 & 192 & 0 & 3 & 48 & 1768 \\
& & & 4 & 0 & 36 & 4 & 48 & 0 \\
& & & 5 & 0 & 8 & 5 & 192 & 0 \\
& & & & & & 6 & 0 & 36 \\
& & & & & & 7 & 0 & 8 \\
\hline
\end{tabular}

Table 2. Clusters obtained for DS2 with $\beta_{0}=0.8182 ; \beta_{0}=0.8636 ; \beta_{0}=0.9091 ;$ and $\beta_{0}=0.9545$

\begin{tabular}{|c|c|c|c|c|c|c|c|c|c|c|c|}
\hline \multicolumn{3}{|c|}{$\beta_{0}=0.8182, \mathrm{AlV}=0.2238$} & \multicolumn{3}{|c|}{$\beta_{0}=0.8636, \mathrm{AIV}=0.2273$} & \multicolumn{3}{|c|}{$\beta_{0}=0.9091$, AIV $=0.2208$} & \multicolumn{3}{|c|}{$\beta_{0}=0.9545, A 1 V^{\prime}=0.2115$} \\
\hline $\mathrm{CN}$ & $\mathrm{NE}$ & NP & $\mathrm{CN}$ & $\mathrm{NE}$ & NP & $\mathrm{CN}$ & $\mathrm{NE}$ & $\mathrm{NP}$ & $\mathrm{CN}$ & $\mathrm{NE}$ & $\mathrm{NP}$ \\
\hline 1 & 2848 & 808 & 1 & 896 & 448 & 1 & 0 & 256 & 1 & 0 & 256 \\
\hline 2 & 768 & 0 & 2 & 768 & 0 & 2 & 704 & 0 & 2 & 512 & 0 \\
\hline 3 & 0 & 1296 & 3 & 1728 & 0 & 3 & 768 & 0 & 3 & 768 & 0 \\
\hline 4 & 0 & 1728 & 4 & 0 & 1296 & 4 & 96 & 0 & 4 & 96 & 0 \\
\hline 5 & 48 & 0 & 5 & 0 & 288 & 5 & 96 & 0 & 5 & 96 & 0 \\
\hline 6 & 48 & 0 & 6 & 192 & 0 & 6 & 1728 & 0 & 6 & 192 & 0 \\
\hline 7 & 0 & 32 & 7 & 0 & 1728 & 7 & 0 & 1296 & 7 & 1728 & 0 \\
\hline 8 & 0 & 8 & 8 & 48 & 0 & 8 & 0 & 192 & 8 & 0 & 1296 \\
\hline 9 & 192 & 0 & 9 & 32 & 72 & 9 & 0 & 288 & 9 & 0 & 192 \\
\hline 10 & 288 & 0 & 10 & 48 & 0 & 10 & 192 & 0 & 10 & 0 & 288 \\
\hline 11 & 0 & 36 & 11 & 0 & 32 & 11 & 0 & 1728 & 11 & 192 & 0 \\
\hline 12 & 0 & 8 & 12 & 0 & 8 & 12 & 48 & 0 & 12 & 0 & 1728 \\
\hline \multirow[t]{11}{*}{13} & 16 & 0 & 13 & 192 & 0 & 13 & 32 & 72 & 13 & 48 & 0 \\
\hline & & & 14 & 288 & 0 & 14 & 48 & 0 & 14 & 0 & 72 \\
\hline & & & 15 & 0 & 36 & 15 & 0 & 32 & 15 & 48 & 0 \\
\hline & & & 16 & 0 & 8 & 16 & 0 & 8 & 16 & 0 & 32 \\
\hline & & & 17 & 16 & 0 & 17 & 192 & 0 & 17 & 0 & 8 \\
\hline & & & & & & 18 & 288 & 0 & 18 & 192 & 0 \\
\hline & & & & & & 19 & 0 & 36 & 19 & 288 & 0 \\
\hline & & & & & & 20 & 0 & 8 & 20 & 32 & 0 \\
\hline & & & & & & 21 & 16 & 0 & 21 & 0 & 36 \\
\hline & & & & & & & & & 22 & 0 & 8 \\
\hline & & & & & & & & & 23 & 10 & 0 \\
\hline
\end{tabular}




\section{Conclusions}

The method proposed in this paper allows obtaining a well-defined clustering, based in an intra-cluster similarity criterion.

The method gives a threshold value $\beta_{0}$ to obtain a well-defined clustering for large data sets.

The method does not establish any assumptions about shape, size or cluster density characteristics of the resultant clusters in each generated clustering. However, the proposed method is still susceptible to noise.

Our method uses the $\beta_{0}$-connected component criterion for clustering. As future work we will work in the generalization of the proposed Intra_ $i$ criterion in order to handle other clustering criteria as those exposed in [4].

Acknowledgement. This work was financially supported by CONACyT (Mexico) through project J38707-A.

\section{References}

1. Duda R., Hart P., and Stork D.: Pattern Classification (2nd ed). Wiley, New York, NY (2000)

2. Martínez-Trinidad J. F. and Guzmán-Arenas A.: The logical combinatorial approach to pattern recognition an overview through selected works. Pattern Recognition 34(4) (2001) $741-751$

3. Ruiz-Shulcloper J. and Mongi A.: A. Logical Combinatorial Pattern Recognition: A Review. In Recent Research Developments in Pattern Recognition, Ed. Pandalai, Pub. Transword Research Networks, USA. (To appear)

4. Martinez Trinidad J. F., Ruiz Shulcloper J., and Lazo Cortes M.: Structuraliation of universes. Fuzzy Sets and Systems. Vol. 112 No. 3 (2000) 485-500

5. Sanchez-Diaz G. and Ruiz-Shulcloper J.: MID mining: a logical combinatorial pattern recognition approach to clustering large data sets. Proc. 5th Iberoamerican Symposium on Pattern Recognition, Lisbon, Portugal (2000) 475-483

6. Pico Peña R.: Determining the similarity threshold for clustering algorithms in the Logical Combinatorial Pattern Recognition through a dendograme. Proc. $4^{\text {th }}$ Iberoamerican Simposium of Pattern Recognition. Havana Cuba (1999) 259-265

7. Reyes Gonzales R. and Ruiz-Shulcloper J.: An algorithm for restricted structuralization of spaces. Proc. $4^{\text {th }}$ Iberoamerican Simposium of Pattern Recognition. Havana Cuba (1999) 267-278

8. Ruiz-Shulcloper J. and Montellano-Ballesteros J.: A new model of fuzzy clustering algorithms. Proc. of the $3^{\text {rd }}$ EUFIT, Aachen, Germany (1995) 1484-1488

9. Ruiz-Shulcloper J., Sanchez-Diaz G. and Abidi M.: Clustering Mixed Incomplete Data. Heuristics \& Optimization for Knowledge Discovery. Idea Group Publishing, USA (2002) 88-106

10. Han J. and Kamber M.: Data mining: concepts and techniques. The Morgan Kaufmann Series in Data Management Systems, Jim Gray Series Editor (2000)

11. Blake C.L. and Merz, C.J.: UCI Repository of machine learning databases [http://www.ics.uci.edu/ mlearn/MLRepository.html]. Irvine, CA: University of California, Department of Information and Computer Science (1998) 